Title: Asymmetrical Reasons, Newborn Infants and Resource Allocation

Authors: Dean Hayden ${ }^{1,2}$, Dominic Wilkinson ${ }^{2,3}$

Affiliations:1. Faculty of Medicine, Nursing, and Health Sciences, Monash University, Australia 2. Oxford Uehiro Centre for Practical Ethics, Faculty of Philosophy, University of Oxford, UK. 3. John Radcliffe Hospital, Oxford, UK.

Correspondence: Prof Dominic Wilkinson, Oxford Uehiro Centre for Practical Ethics, Suite 8, Littlegate House, St Ebbes St, Oxford, OX1 1PT, UK. Tel: +44 1865 286888, Fax: +44 1865 286886 Email: dominic.wilkinson@philosophy.ox.ac.uk

Dominic Wilkinson Bio: Dominic Wilkinson is Director of Medical Ethics and Professor of Medical Ethics at the Oxford Uehiro Centre for Practical Ethics, University of Oxford. He is a consultant in newborn intensive care at the John Radcliffe Hospital, Oxford. Dominic has published more than 100 academic articles relating to ethical issues in intensive care for adults, children and newborn infants. He is the author of 'Death or Disability? The 'Carmentis Machine' and decision-making for critically ill children' (Oxford University Press 2013).

Dean Hayden Bio: Dean Hayden is a Bachelor of Medicine/Bachelor of Surgery (MBBS) student at Monash University in Melbourne, Australia. He is currently undertaking research in Bioethics at the Oxford Uehiro Centre for Practical Ethics, focusing on resource allocation for resuscitation of preterm infants in low and middle-income countries.

Funding: DW was supported for this work by a grant from the Wellcome trust WT106587/Z/14/Z

The authors declare no competing interests.

ORCID ID:

DW: 0000-0003-3958-8633 
In his target article "Saving or Creating - Which are we doing when we resuscitate extremely preterm infants?", Travis Rieder argues that asymmetrical reasons would justify withholding resuscitation from some Extremely Preterm Infants(EPIs) when this would not be justified in older children.(Rieder 2017)

Our commentary focuses on two issues raised by the target article. First, we critically analyze the normative basis of The Asymmetry and when it should apply to decisions. Second, we look at the wider implications of asymmetrical reasons for resource allocation.

\section{THE ASYMMETRY}

Rieder's argument about the risks and benefits of life-sustaining treatment (LST) for EPIs is based on the force of reasons to bring or not bring an individual into existence. He draws on a pair of widely shared, but apparently asymmetrical intuitions:

1. there is at most a relatively weak moral reason to bring a child into the world who will have a life of overall benefit (a life worth living LWL);

2. there is a strong moral reason not to bring a child into the world who will have a life of net suffering (a life not worth living LNWL).

Rieder calls these "creative reasons"(2017); Philosophers have called this 'The Asymmetry.' (McMahan 2009) Because of The Asymmetry, in making decisions about bringing children into the world, we should give much more weight to the possibility of harm, than to the possibility of benefit. In contrast, for an older child, there is both a strong moral reason to save their life if they will have a LWL, and a strong moral reason not to prolong their life if they would have a LNWL; we should give similar weight to the harms and benefits of lifeprolonging medical treatment (Rieder calls these "rescue reasons").

Rieder applies the asymmetry to EPIs through his claim that there is a "Creative spectrum" extremely preterm infants near the borderline of viability are still in the process of being created. Consequently, our reasoning about risks and benefits of treatment should lie somewhere in between creation and rescue.

However, beyond appealing to their small size and physical immaturity, Rieder doesn't clearly articulate why creative reasons apply to EPIs. There are two problems with this. On the one hand, some will reject the idea of a creative spectrum. This might be motivated by a religious belief that human life is created by a divine being, or a non-religious intuitive belief that infants are created at the point of conception. On the other hand, physical and cognitive development continues for a long period after a child is born (even at full term). For example, postnatal neural development includes substantial proliferation and migration of glial progenitors, myelination, synapse formation and evolution of neural networks, while the brain increases in size four-fold during the pre-school period.(Stiles and Jernigan 2010) If this is right, creative reasons might apply well into childhood.

To assess the scope of asymmetrical reasons, we need a clearer understanding of their basis. At its most fundamental, The Asymmetry arises because of the existence or nonexistence of harms. Noone is harmed if parents decide not to bring a child into the world, but someone can be harmed if they are brought into the world and have a LNWL. This means that harms apply only in one direction for conception decisions.

The Asymmetry doesn't apply in that way to a newborn infant. A newborn infant can be harmed if they die (McMahan 2002, 352-3; Wilkinson 2013, 128-133) and they can be harmed if they survive. Harms apply in both directions. However, one of us (DW) has previously argued that those harms have unequal weights.(ibid, 133-145) The harm of death 
may be a lesser harm than it would be for an older child. That is because a newborn has relatively weak psychological links to his or her future.(McMahan 2002, 352) They have limited awareness of themselves, have few memories, have not yet established desires, plans and hopes for their future.

If the harm of death is relatively reduced for a newborn, that would support Rieder's suggestion that we think about decisions for EPIs differently from the way that we think about decisions for older children. Importantly, though, this explanation of asymmetrical reasoning isn't restricted to physically immature premature infants. Where there is a significant risk that a newborn infant may be harmed by life-sustaining treatment, parents' views about whether to provide treatment should be sought and respected.(Wilkinson 2010) This would apply to any newborn infant with an uncertain, but potentially poor prognosis, regardless of their gestation.

\section{RESOURCE ALLOCATION}

Rieder focuses on the risks versus benefit for individual infants. However, if asymmetrical reasons apply, and if we are morally justified in placing less weight on the future benefits of treatment for extremely preterm infants (than for older children), this may have wider implications for resource allocation decisions. It may imply preferentially directing resources to older infants or children.

For example, cost-effectiveness analysis in public healthcare systems often assesses the cost per Quality-Adjusted-Life-Year (QALY) in order to compare interventions. The underlying assumption is that all QALYs are of equal social value, irrespective of which individual accrues them.(Whitehead and Ali 2010) Neonatal intensive care, even at low gestational age, is often regarded as more cost effective than intensive care for older patients. This is explained by the fact that: (i) doomed infants die early; (ii) the smallest and sickest die the quickest; and (iii) survivors live a long time after discharge, so high initial costs are spreadout over a lifetime.(Meadow and Lantos 2009) However, if there is a weaker moral reason to save the life of an EPI, there appears to be less reason to allocate resources to such an infant. Rieder's spectrum appears to imply less weight to QALYs gained by an EPI compared to an older child or adult. That may affect whether or when treatment of EPIs would be cost-effective.

Some have challenged the application of cost-effectiveness to resource allocation.(Whitehead and Ali 2010) Yet the same implications arise if looking purely at costs. Under any healthcare system, there will be a maximum amount which the system (insurer, or public healthcare) is prepared to spend on treatment. Let us call this amount $\$ X$. If Rieder is correct that we should "discount our rescue reasoning" for an EPI, that would yield a cost-threshold spectrum that would increase with increasing gestation. Furthermore, given an inverse relationship between treatment costs and the gestational age of the infant (Mangham et al. 2009) there may be a degree of prematurity where the costs of resuscitation and intensive care become greater than the relative cost-threshold (Figure 1). 


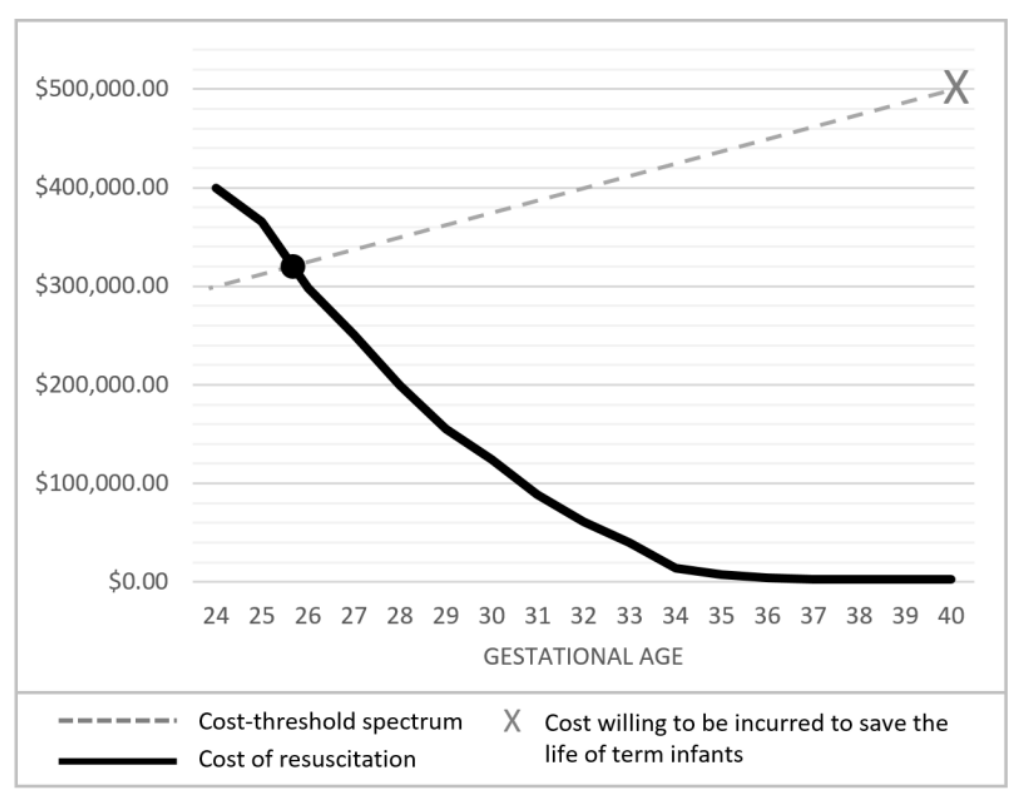

\section{Figure 1 - The 'Cost-threshold' spectrum - A schematic representation of the implications of Rieder's 'Creative-spectrum' for the affordability of treatment for preterm infants. ${ }^{1}$}

Asymmetrical reasons will not necessarily rule out treatment of EPIs. Some well-resourced healthcare systems may place the value of $X$ to be so high that, even if discounted, resuscitation of extremely preterm infants would be justified. Yet this is unlikely to be the case in the majority of the world's low and middle-income countries (LMICs). Whilst there is very little evidence in these countries as to the actual costs of newborn resuscitation, we could reasonably expect that Rieder's views would preclude resuscitation at very low gestational ages in such settings.

What would the implications be of asymmetrical reasons for funding of neonatal intensive care in resource limited settings? The World Health Organization's Choosing Interventions that are Cost-Effective (WHO-CHOICE) initiative makes recommendations on resource allocation that are applicable to LMICs. It has suggested that countries consider an intervention to be 'cost-effective' when, per Disability-Adjusted-Life Year averted, it costs less than three times the country's Gross Domestic Product per capita.(Marseille et al. 2015) If we incorporate Reider's spectrum view, that would imply that the WHO-CHOICE initiative should be modified to employ varying proportions of GDP per capita relative to gestational age when evaluating the cost-effectiveness of newborn resuscitation.

For example, a country such as El Salvador has a GDP per capita of US\$4,219. According to WHO-CHOICE, the Salvadorian healthcare system should be willing to spend at most US\$12,657/DALY. However, if the benefits of treatment should be discounted for EPIs, or more generally for newborn infants, this maximum threshold should be reduced by at least some degree. Currently Salvadoran clinicians generally do not resuscitate infants born prior to 26 weeks' gestational age, partly due to costs.(Martinez et al. 2009) However, depending on the costs of neonatal intensive care in this setting, and the degree of discounting,

\footnotetext{
${ }^{1}$ Costs of resuscitation (Phibbs and Schmitt 2006) have been adjusted for inflation to \$US 2017 according to the Consumer Price Index (Bureau of Labor Statistics 2017). For illustration purposes, the cost threshold spectrum has been constructed on the assumption of a linear trend directly proportional to gestational age. This potentially implies that at 20 weeks' gestation, there is half the reason to rescue, and therefore half the reason to spend.
} 
Rieder's view may suggest that clinicians adopt a later resuscitation threshold than is currently applied.

Rieder's target article highlights that treatment of critically ill infants offers great benefit, but may also harm infants and incur enormous costs - as in the case example that he cites.(Rieder 2017) There are reasons to evaluate the risks and benefits of treatment somewhat differently for newborn infants compared to older children. We have argued that those reasons are not confined to extremely preterm infants. We have also highlighted the challenging implications of asymmetrical reasons for resource allocation in neonatal intensive care. While wealthy countries may be able to provide such highly expensive treatment without apparent limit, other countries do not have that luxury and must decide whether and when to allocate scarce resources to saving newborn infants.

\section{References:}

Bureau of Labor Statistics. 2017. CPI Inflation Calendar. Available at: https://www.bls.gov/data/inflation_calculator.htm. (Accessed May 11, 2017).

Mangham, L. J., S. Petrou, L. W. Doyle, E. S. Draper, and N. Marlow. 2009. The cost of preterm birth throughout childhood in England and Wales. Pediatrics. 123(2):312-27.

Marseille, E., B. Larson, D. S. Kazi, J. G. Kahn, and S. Rosen. 2015. Thresholds for the costeffectiveness of interventions: alternative approaches. Bulletin of the World Health Organization. 93(2):118-124.

Martinez, A. M., E. D. Mathes, A. F. Foster-Rosales, and J. C. Partridge. 2009. Obstetricians' attitudes and practices of life support for extremely premature low birth weight infants in El Salvador. Journal of neonatal-perinatal medicine. 2(1):49-56.

McMahan, J. 2002. The ethics of killing: problems at the margins of life. New York: Oxford University Press.

McMahan, J. 2009. Asymmetries in the Morality of Causing People to Exist. In Harming Future Persons: Ethics, Genetics and the Nonidentity Problem, ed. M. A. Roberts, and D. T. Wasserman, 49-68. Dordrecht: Springer Netherlands.

Meadow, W., and J. Lantos. 2009. Moral reflections on neonatal intensive care. Pediatrics. 123(2):595-7.

Phibbs, C. S., and S. K. Schmitt. 2006. Estimates of the cost and length of stay changes that can be attributed to one-week increases in gestational age for premature infants. Early human development. 82(2):85.

Rieder, T. 2017. Saving or Creating - Which are we doing when we resuscitate extremely preterm infants? American Journal of Bioethics

Stiles, J., and T. L. Jernigan. 2010. The Basics of Brain Development. Neuropsychology Review. 20(4):327. 
Whitehead, S. J., and S. Ali. 2010. Health outcomes in economic evaluation: the QALY and utilities. British Medical Bulletin. 96(1):5-21.

Wilkinson, D. 2010. A life worth giving: the threshold for permissible withdrawal of treatment from disabled newborn infants. American Journal of Bioethics. 11(2):20-32.

Wilkinson, D. 2013. Death or Disability?: The 'Carmentis Machine' and Decision-Making for Critically III Children. Oxford: Oxford University Press. 